\title{
Article \\ Simulation Study of Silicon-Based Single-Photon Avalanche Diodes with Double Buried Layers and Deep Trench Electrodes
}

\author{
Yanyan $\mathrm{Du}^{1}{ }^{1} \mathrm{Bo} \mathrm{Li}^{1, *}$ and $\mathrm{Xu}$ Wang ${ }^{2}$ \\ 1 College of Nuclear Equipment and Nuclear Engineering, Yantai University, No.30 Qingquan Road, \\ Yantai 264005, China; duyy@ytu.edu.cn \\ 2 School of Intelligent Engineering, Shandong Management University, No.3500 Dingxiang Road, \\ Jinan 250357, China; wangxu@sdmu.edu.cn \\ * Correspondence: boli@ytu.edu.cn; Tel.: +86-152-6658-1347
}

Citation: Du, Y.; Li, B.; Wang, X. Simulation Study of Silicon-Based Single-Photon Avalanche Diodes with Double Buried Layers and Deep Trench Electrodes. Crystals 2021, 11, 1176. https://doi.org/10.3390/ cryst11101176

Academic Editors: M. Ajmal Khan and Alessandro Chiasera

Received: 25 August 2021

Accepted: 24 September 2021

Published: 28 September 2021

Publisher's Note: MDPI stays neutral with regard to jurisdictional claims in published maps and institutional affiliations.

Copyright: (c) 2021 by the authors. Licensee MDPI, Basel, Switzerland. This article is an open access article distributed under the terms and conditions of the Creative Commons Attribution (CC BY) license (https:// creativecommons.org/licenses/by/ $4.0 /)$.

\begin{abstract}
In this paper we present a study of a silicon-based Single-Photon Avalanche Diode (SPAD) in the near-infrared band with double buried layers and deep trench electrodes fabricated by the complimentary metal-oxide semiconductor (CMOS) technology. The deep trench electrodes aim to promote the movement of carriers in the device and reduce the transit time of the photo-generated carrier. The double buried layers are introduced to increase the electric field in the avalanche area and withstand a larger excess bias voltage as its larger depletion region. The semiconductor device simulation software TCAD is used to simulate the performance of this SPAD model, such as the I-V characteristic, the electric field and the Photon Detection Efficiency (PDE). Further optimization of the structure are studied with influence factors such as the doping concentration and depletion region thickness. Based on the results in this study, the designed a structure that can provide a high detecting efficiency in the near-infrared band.
\end{abstract}

Keywords: SPAD; double buried layers; trench electrodes; PDE

\section{Introduction}

The Single-Photon Avalanche Diode (SPADs) has become one of the most crucial photodetectors due to its high sensitivity in light detecting [1,2]. Based on tre avalanche effect, even a weak optical signal can produce a large current in the SPAD due to its high gain, high signal-to-noise ratio, low dark count, high sensitivity and fast response. Since Silicon-based SPAD (Si-SPAD) is compatible with CMOS circuits and produced with a low manufacturing cost and mature technology, it has great advantages in optical fiber communication, lidar and other fields involving weak light detection [3,4]. However, the low detecting efficiency in the near-infrared band limits its further application.

To increase the Photon Detection Efficiency (PDE) in the near-infrared band, many studies have been performed around the world, examining approaches such as introducing guard ring structures, tuning the multiplication region depth and so on [5-7]. Here, we introduce a new structure with double buried layers and deep trench electrodes. The buried layers are used to adjust the distribution of the avalanche and depletion region, and realize the uniform distribution of the electric field [8-10]. The deep trench electrodes together with the P-buried layer can form a low resistance path for the carrier transmission between electrodes and improve the internal electric field of the device.

The detailed information of the structure is described in Section 2. Then, the study of the performance of this SPAD is detailed in Section 3. Based on the study results, the final conclusion is given in Section 4.

\section{Simulations}

The simulated software is TCAD, which is widely used in the simulation of new semiconductor processes and device characteristics. The numerical calculation in TCAD is 
based on grid computing with a series of physical models and related equations, which are based on the mature theories of solid state physics and semiconductor physics or some empirical formulas. The physical models in our study include concentration-dependent mobility (conmob), field-dependent mobility (fldmob), Shockley-Read-Hall recombination (srh), Auger recombination (auger), band-to-band tunneling (bbt.std), trap-assisted tunneling (TRAP.TUNNEL) and the ray tracing model (optr).

The simulated 2D structure of this SPAD is shown in Figure 1 below, consisting of a Si wafer with a thickness of $10 \mu \mathrm{m}$. The SPAD mainly consists of a p-type substrate, a $\mathrm{p}$ well, a p-type buried layer, an n-type buried layer, $\mathrm{N}-$, an $\mathrm{N}$ well, an $\mathrm{N}+$ region, a deep trench anode and shallow trench isolation (STI). The STIs are introduced to prevent lateral diffusion of doping in the active region and make the electric field more uniform. Differently from the traditional device with a P+/Deep n well (DNW) junction, the avalanche junction is formed by the p-type buried layer and n-type buried layer. The p-type buried layer is buried on the p-type substrate and the n-type buried layer is used to adjust the depletion layer width and electric field. The depletion and light absorption region lie between the $\mathrm{N}$ well and n-type buried layer. Additionally, two deep trench anodes are contacted with the p-type buried layer on both sides. The values and distributions of doping concentration for each region are shown in Table 1 and Figure 2. The electric field distribution of the simulated structure with the over-voltage $\left(V_{O V}\right)$ is 1.3 times the breakdown voltage $\left(V_{B R}\right)$, and is shown in Figure 3a.The width of the depletion and absorption region is large enough to ensure the absorption performance of incoming photons, and the maximum electric field in the multiplication region is large than $5 \times 10^{5} \mathrm{~V} / \mathrm{cm}$, as shown in Figure $3 \mathrm{~b}$.

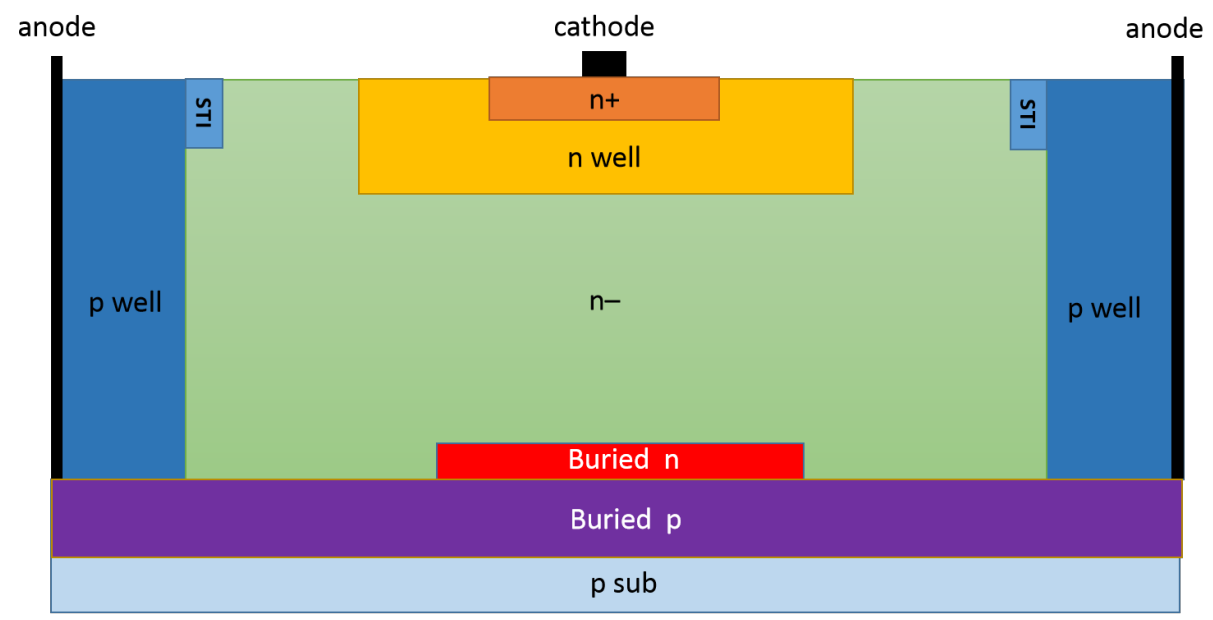

Figure 1. Cross section of the SPAD structure.

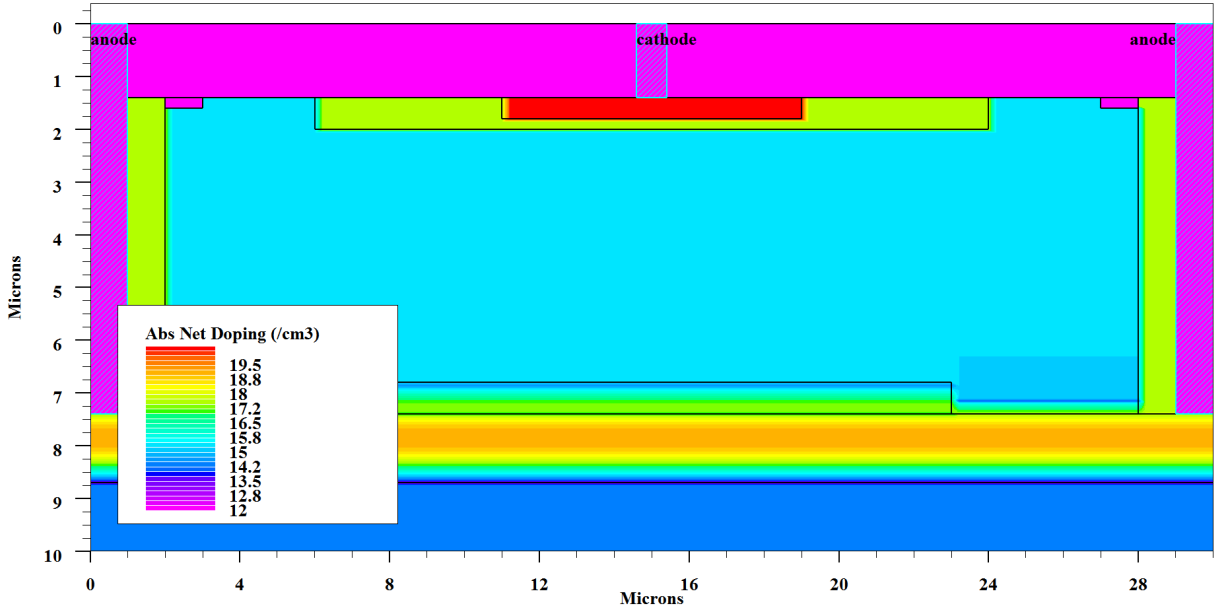

Figure 2. Doping concentration for each region in the simulated cross section of the structure. 
Table 1. Doping concentration for each region.

\begin{tabular}{lcc}
\hline Region & Value $\left(/ \mathrm{cm}^{\mathbf{3}}\right)$ & Type \\
\hline $\mathrm{N}+$ & $1 \times 10^{20}$ & uniformity \\
$\mathrm{N}$ Well & $2 \times 10^{17}$ & uniformity \\
$\mathrm{N}-$ & $1 \times 10^{15}$ & uniformity \\
P well & $2 \times 10^{17}$ & uniformity \\
N buried layer & $2 \times 10^{17}$ & gauss \\
P buried layer & $1 \times 10^{19}$ & gauss \\
\hline
\end{tabular}

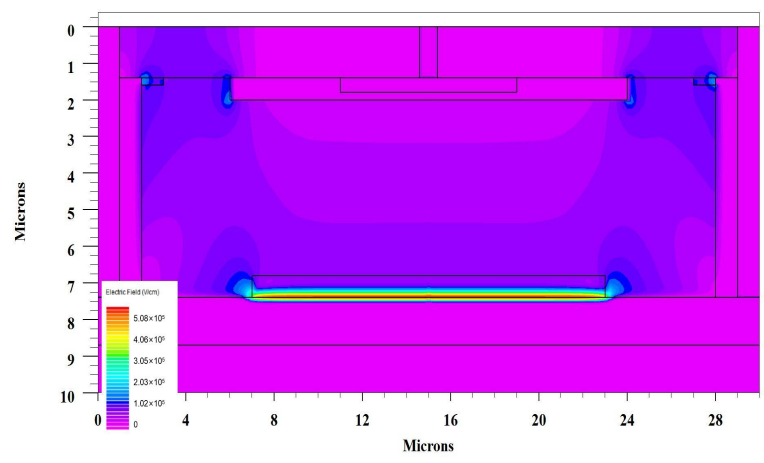

(a)

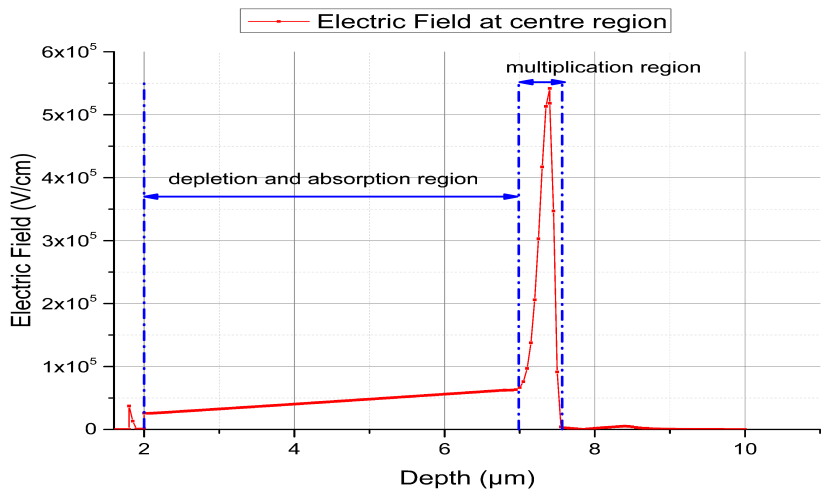

(b)

Figure 3. The electric field in the simulated structure. (a) Electric field distribution in 2D simulated structure. (b) Electric field in the central region $(x=15 \mu \mathrm{m})$.

\section{Result and Discussion}

Since the SPAD works in Geiger mode, in order to explore the performance of the SPAD, the breakdown voltage $\left(V_{B R}\right)$ must be determined first. The breakdown voltage is an important parameter used to measure the reliability and application range of the whole device. An exorbitant breakdown voltage will shorten the service life of the device. The breakdown voltage is mainly related to the doping concentration on both sides of the PN junction. In this paper, the $V_{B R}$ is defined as the voltage value at the current of $10 \mu \mathrm{A}$ to prevent confusion between various breakdown voltage definitions among different studies.

Another important parameter is the Photon Detection Efficiency (PDE) of the SPAD. The PDE can be calculated by the equation below [11]:

$$
\operatorname{PDE}\left(\lambda, V_{O V}\right)=\eta(\lambda) \times P_{t r}\left(V_{O V}\right),
$$

where $\eta(\lambda)$ is the quantum efficiency with the light wavelength $\lambda$, and $P_{t r}\left(V_{O V}\right)$ is the avalanche-triggering probability for a certain over-voltage $\left(V_{O V}\right) . \eta(\lambda)$ is an important parameter used to characterize the photoelectric conversion efficiency of photoelectric devices, and it is defined as the ratio of the number of generated electron hole pairs to the number of incident photons. In the TCAD simulation, this value can be calculated by the ratio of the anode current to the source photon current. $P_{t r}\left(V_{O V}\right)$ is equal to the ratio of the number of avalanches triggered by carriers to the total number of photogenerated carriers, and can be directly retrieved from the variable referring to probability of avalanche joint in the TCAD simulation.

\subsection{Deep Trench Anode}

The usage of deep trench electrodes in this structure is inspired by reference [12]. It is proposed to apply trench electrodes to optimize the current distribution in the device and improve the sensitivity of the whole device. The comparison of the performance of deep trench anodes together with anodes at the surface and half into the device is 
shown in Figure 4. Figure 4a-c are the current density for three different designs when the over-voltage $\left(V_{O V}\right)$ is 1.3 times the breakdown voltage $\left(V_{B R}\right)$. As shown in these figures, the deep trench anodes (anodes contacted with the $\mathrm{P}$ buried layer) can improve the current distribution and further reduce photocarrier transit time since the deep trench anodes are directly in contact with the $p$ buried layer. Thus, the device with deep trench anodes can reach the anode current of $10 \mu \mathrm{A}$ at a lower $V_{B R}$. The I-V characteristics of three devices are shown in Figure $4 \mathrm{~d}$. The device with deep trench anodes has a smaller breakdown voltage, and the saturation current is around $10^{-3} \mathrm{~A}$, which is at least five times better than the others.

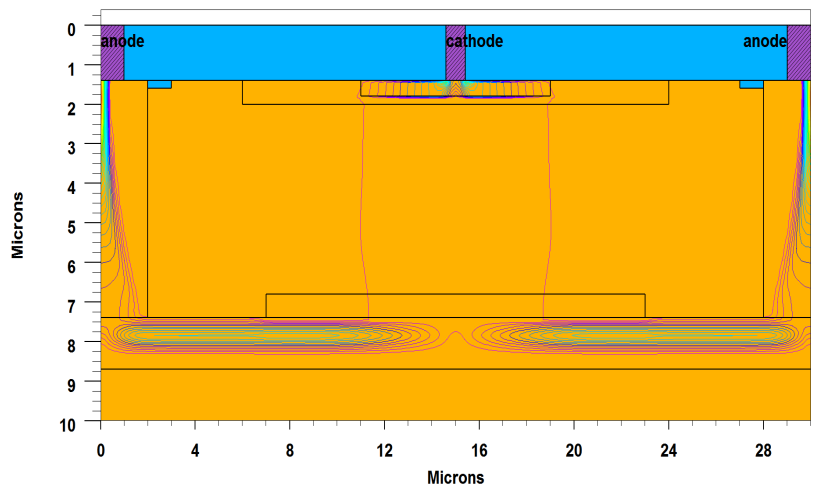

(a)

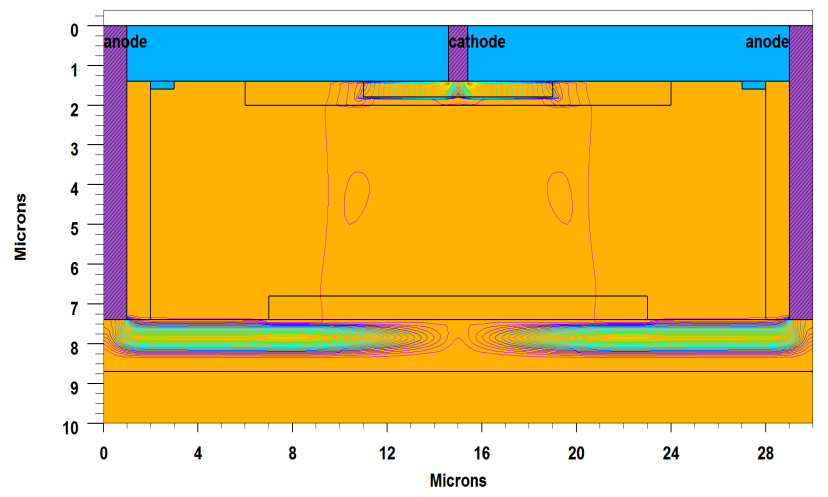

(c)

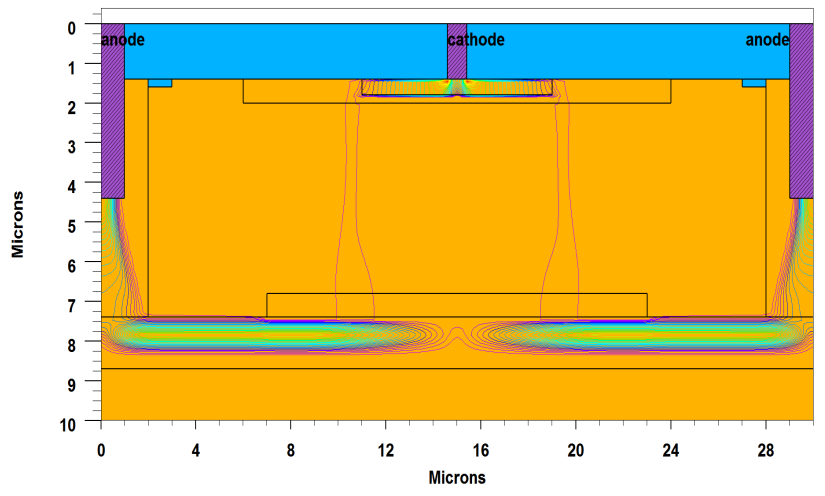

(b)

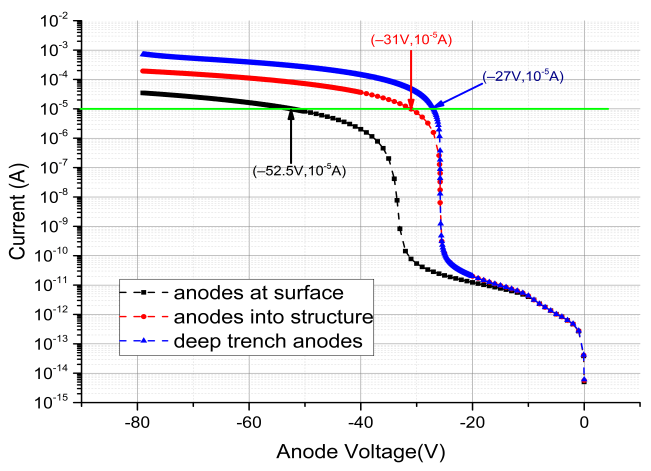

(d)

Figure 4. Comparison of the performance of deep trench anodes together with anodes at the surface and half into the device. (a) Anodes at surface. (b) Anodes inside structure. (c) Deep trench anodes (anodes contacted with P buried layer). (d) $I-V$ for three different designs of anodes.

\subsection{Effect of Depletion Region Thickness}

The region of light absorption is proportional to the area of the depletion region, which will affect the quantum efficiency and then the final PDE. Here we chose several thickness values, and studied the effect on the final PDE. The PDEs' distribution for depletion region thicknesses of $2.5 \mu \mathrm{m}, 3.5 \mu \mathrm{m}, 4.5 \mu \mathrm{m}$ and $5.5 \mu \mathrm{m}$ with light wavelength are shown in Figure 5a. As shown in Figure 5a, the PDE of light above $770 \mathrm{~nm}$ increases when the thickness increases. However, the PDE around 450-600 nm has an obvious rapid decline. The illustration in Figure 5a shows the values of PDE for those thickness at wavelength of 870 and $550 \mathrm{~nm}$. As described in Equation (1), the PDE is the product of the quantum efficiency $(\eta(\lambda))$ and avalanche triggering probability $\left(P_{t r}\left(V_{O V}\right)\right)$. Figure $5 \mathrm{~b}$ shows that $P_{t r}\left(V_{O V}\right)$ will decrease with a thickness increase, whereas Figure $5 \mathrm{c}$ shows that the trends of $\eta(\lambda)$ at different wavelength region are not consistent. The differences of $\eta(\lambda)$ below $600 \mathrm{~nm}$ are small among different thicknesses, which leads the values of PDE around 
450-600 $\mathrm{nm}$ to decrease with a thickness increase, combining the effect from $P_{t r}\left(V_{O V}\right)$. On the contrary, the values of $\eta(\lambda)$ above $600 \mathrm{~nm}$ will increase as the thickness increases, which leads the values of the PDE to increase slightly with a thickness increase, combining the effect from $P_{t r}\left(V_{O V}\right)$. In order to avoid too much of a decrease in visible light detection efficiency, the thickness of about $5.5 \mu \mathrm{m}$ was finally selected.

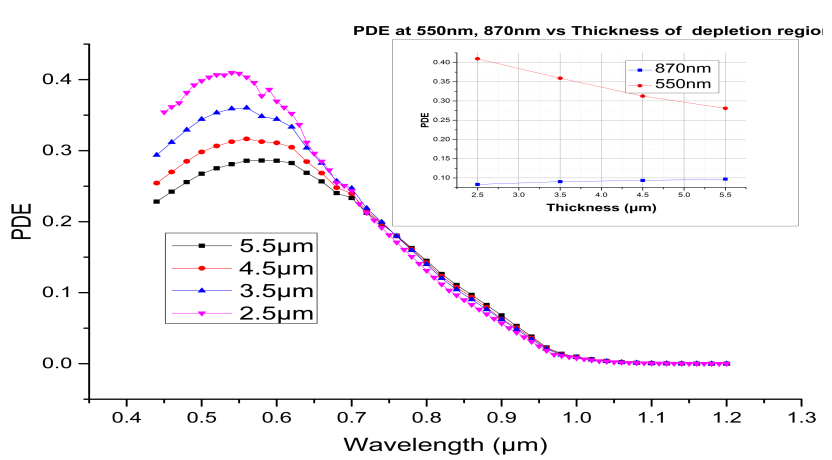

(a)

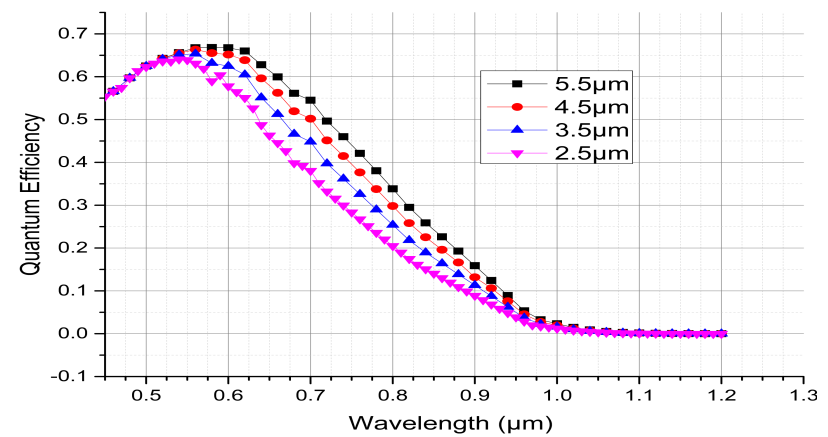

(b)

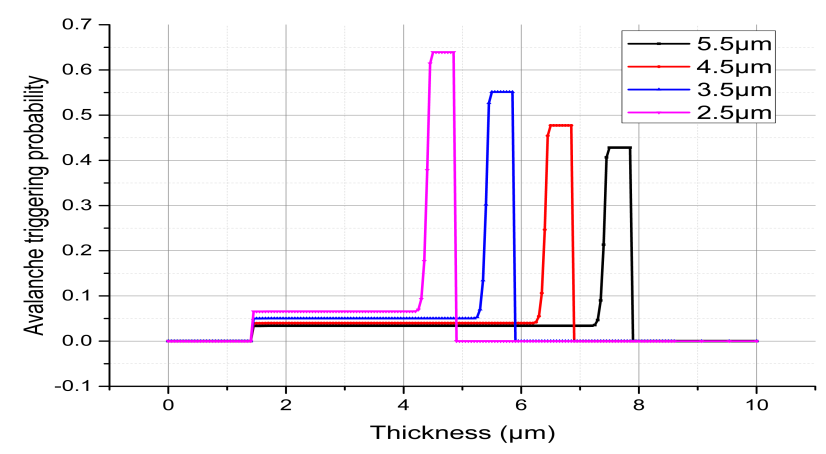

(c)

Figure 5. The PDE performance vs. thickness. (a) PDEs for different depletion region thicknesses. (b) Quantum efficiency for different thicknesses. (c) Avalanche triggering probability for different thicknesses.

\subsection{Effect of Doping Concentration of N Buried Layer}

Since the avalanche junction is formed by the $\mathrm{P}$ buried layer and $\mathrm{N}$ buried layer, the electric fields of the avalanche region are mainly affected by the doping concentration of the $\mathrm{N}$ buried layer. The avalanche triggering probability is determined by the electric field and further affects the PDE. Here, we optimized the doping concentration by comparing the $\mathrm{I}-\mathrm{V}$ characteristics and PDEs with different doping concentration. As shown in Figure 6a, the lower the $\mathrm{N}$ buried doping concentration is, the higher the PDE. The PDE increases from $7.7 \%$ to $12.9 \%$ as the $\mathrm{N}$ buried doping concentration decreases from $2.1 \times 10^{17} / \mathrm{cm}^{3}$ to $1.9 \times 10^{17} / \mathrm{cm}^{3}$ at the wavelength around $870 \mathrm{~nm}$. The reason is that the avalanche triggering probability decreases when the $\mathrm{N}$ buried dose increases, as illustrated in Figure $6 \mathrm{~b}$, and in fact the larger doping concentration will lead to a lower breakdown voltage. In theory, the PDE can continue to rise with smaller doping concentrations. However, as Figure $6 \mathrm{c}$ displays, the breakdown voltage is a function of the $\mathrm{N}$ buried dose, which indicates that the breakdown voltage will increase when the $\mathrm{N}$ doping concentration decreases. As mentioned at the beginning of this section, a high breakdown voltage will affect the service life of the SPAD. Another issue is that too small a doping concentration of the $\mathrm{N}$ buried layer will make the absorption area and avalanche area ambiguous. The doping concentration of $1.9 \times 10^{17} / \mathrm{cm}^{3}$ with a breakdown voltage $\left(V_{B R}\right)$ around $40 \mathrm{~V}$ is suitable. 


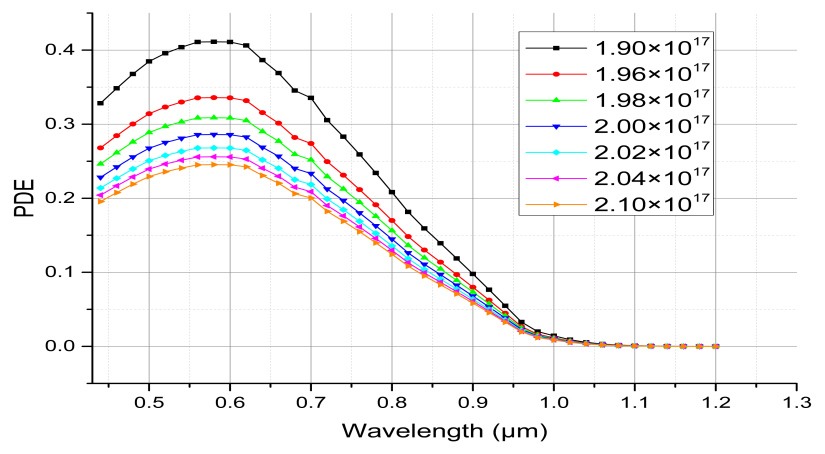

(a)

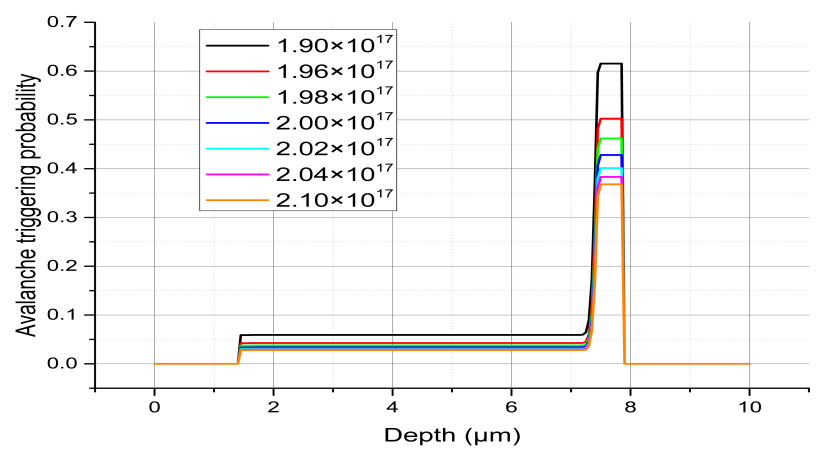

(b)

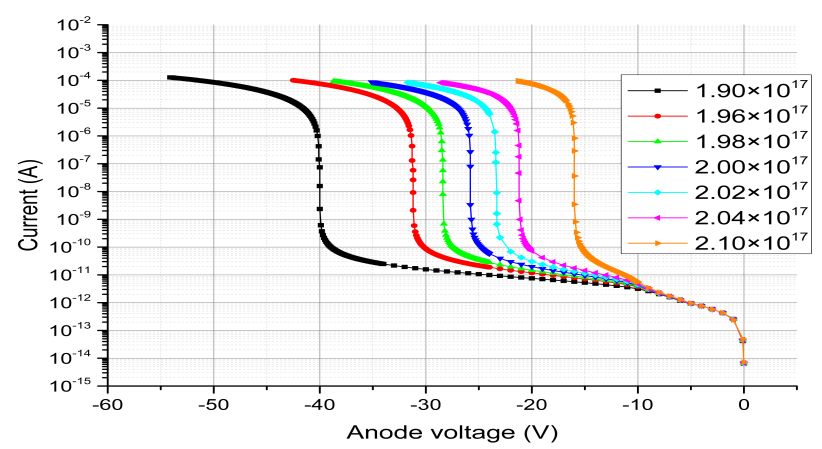

(c)

Figure 6. The performance of different doping concentrations of the $\mathrm{N}$ buried layer. (a) PDEs for different doping concentrations. (b) Avalanche triggering probabilities for different doping concentrations. (c) I-V characteristics for different doping concentrations.

\section{Conclusions}

After the performance study in Sections 3.2 and 3.3, the depletion region thickness of $5.5 \mu \mathrm{m}$ and doping concentration of $1.9 \times 10^{17} / \mathrm{cm}^{3}$ were used in the final device. The breakdown voltage of this structure is $41.6 \mathrm{~V}$ with a $10 \mu \mathrm{A}$ current. The distribution of electric field in the central region of the structure with a over-voltage of $54.1 \mathrm{~V}\left(1.30 V_{B R}\right)$ is shown in Figure 7a. The electric field can be well adjusted, as we expected. As shown in Figure $7 \mathrm{~b}$, the double buried layers and deep trench anodes can achieve a PDE of $12.9 \%$ at $870 \mathrm{~nm}$ with an over-voltage of $54.1 \mathrm{~V}\left(1.30 V_{B R}\right)$, which provides a possible device structure to improve the detection efficiency in the NIR band.

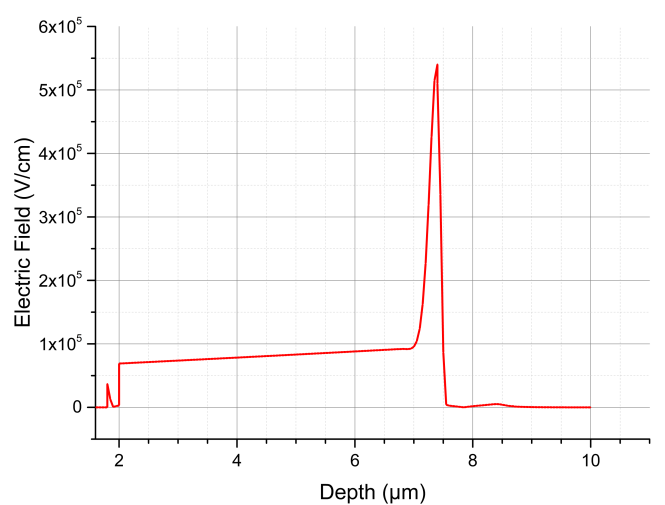

(a)

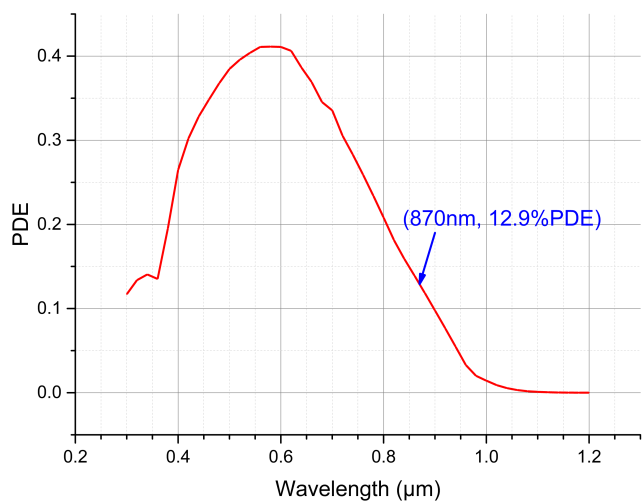

(b)

Figure 7. The electric field and PDE of the final designed structure. (a) Electric field in the central region with an over-voltage of $54.1 \mathrm{~V}\left(1.30 V_{B R}\right)$. (b) PDE distribution vs. light wavelength. 
Author Contributions: Conceptualization, Y.D. and B.L.; methodology, Y.D.; software, Y.D. and B.L.; validation, B.L. and X.W.; investigation, X.W.; data curation, Y.D. and B.L.; writing, Y.D. and B.L.; funding acquisition, Y.D. All authors have read and agreed to the published version of the manuscript.

Funding: This research was funded by the National Natural Science Foundation of China (Grant number 12005181).

Data Availability Statement: The data presented in this study are available upon request from the corresponding author.

Acknowledgments: We thank Xiao Zhao, Meiqin Zhang and Xiangli Qian for their helpful discussion.

Conflicts of Interest: The authors declare no conflict of interest.

\section{References}

1. Campbell, J. Recent Advances in Avalanche Photodiodes. J. Light. Technol. 2016, 34, 278-285. [CrossRef]

2. Steindl, B.; Hofbauer, M.; Schneider-Hornstein, K.; Brandl, P.; Zimmermann, H. Single-photon avalanche photodiode based fiber optic receiver for up to $200 \mathrm{Mb} / \mathrm{s}$. IEEE J. Sel. Top. Quantum Electron. 2017, 24, 1-8. [CrossRef]

3. Gyongy, I.; Al Abbas, T.; Finlayson, N.; Johnston, N.; Calder, N.; Erdogan, A.; Dutton, N.W.; Walker, R.; Henderson, R.K. Advances in CMOS SPAD sensors for LIDAR applications. Emerg. Imaging Sens. Technol. Secur. Def. III 2018, 10799, 1079907.

4. Bruschini, C.; Homulle, H.; Antolovic, I.M.; Burri, S.; Charbon, E. Single-photon avalanche diode imagersin biophotonics: Review and outlook. Light. Sci. Appl. 2019, 8, 1-28. [CrossRef] [PubMed]

5. Faramarzpour, N.; Deen, M.J.; Shirani, S.; Fang, Q. Fully Integrated Single Photon Avalanche Diode Detector in Standard CMOS $0.18 \mu \mathrm{m}$ Technology. IEEE Trans. Electron Devices 2008, 55, 760-767. [CrossRef]

6. Wei, W.; Yu, Z.; Wei, Z. High-Performance Structure of Guard Ring in Avalanche Diode for Single Photon Detection. Int. J. Commun. Netw. Syst. Sci. 2017, 10, 1-6.

7. Richardson, J.A.; Webster, E.; Grant, L.A.; Henderson, R.K. Scaleable Single-Photon Avalanche Diode Structures in Nanometer CMOS Technology. IEEE Trans. Electron Devices 2011, 58, 2028-2035. [CrossRef]

8. Niclass, C.; Matsubara, H.; Soga, M.; Ohta, M.; Ogawa, M.; Yamashita, T. A NIR-Sensitivity-Enhanced Single-Photon Avalanche Diode in $0.18 \mu \mathrm{m}$ CMOS. In Proceedings of the International Image Sensor Workshop, Vaals, The Netherlands, 8-11 June 2015; pp. 8-11.

9. Takai, I.; Matsubara, H.; Soga, M.; Ohta, M.; Ogawa, M.; Yamashita, T. Single-Photon Avalanche Diode with Enhanced NIRSensitivity for Automotive LIDAR Systems. Sensors 2016, 16, 459. [CrossRef] [PubMed]

10. Ping, X.; Yue, X. Improved photon detection efficiency of single photon avalanche diodes with buried layer structure. In Proceedings of the 2016 13th IEEE International Conference on Solid-State and Integrated Circuit Technology (ICSICT), Hangzhou, China, 25-28 October 2016.

11. Pancheri, L.; Stoppa, D.; Betta, G.D. Characterization and Modeling of Breakdown Probability in Sub-Micrometer CMOS SPADs. IEEE J. Sel. Top. Quantum Electron. 2014, 20, 328-335. [CrossRef]

12. Shao, L.; Li, T.; Chen, Y.X.; Wang, Y. Design and Optimization of 500 V Trench Anode LIGBT. J. Beijing Univ. Technol. 2012, 38, 1153-1157. 